\title{
NETs promote liver metastasis via CCDC25
}

CS

\section{NET-DNA}

was found to

substantially

promote the

migration and

adhesion of

cancer cells
Preclinical evidence suggests that neutrophil extracellular traps (NETs) might have a role in cancer metastasis. Now, a study published in Nature has shown that NETs are abundant in the liver metastases of patients with colon cancer and that they act as a chemotactic factor attracting cancer cells. This study also identified a receptor on cancer cells mediating NET-associated metastasis that could be therapeutically targeted.

Neutrophils are a key effector of the innate immune system and one of their functions is the production of NETs to trap microorganisms during infection. NETs are extracellular DNA structures composed of decondensed chromatin filaments, and excessive NET formation can have pro-inflammatory characteristics that can contribute to cell and host injury. Indeed, NETs have been proposed to play a part in a range of diseases in various organs.

In studies of cancer metastasis, work in mice has shown that NETs facilitated metastasis in the liver and the lung. In accordance with their

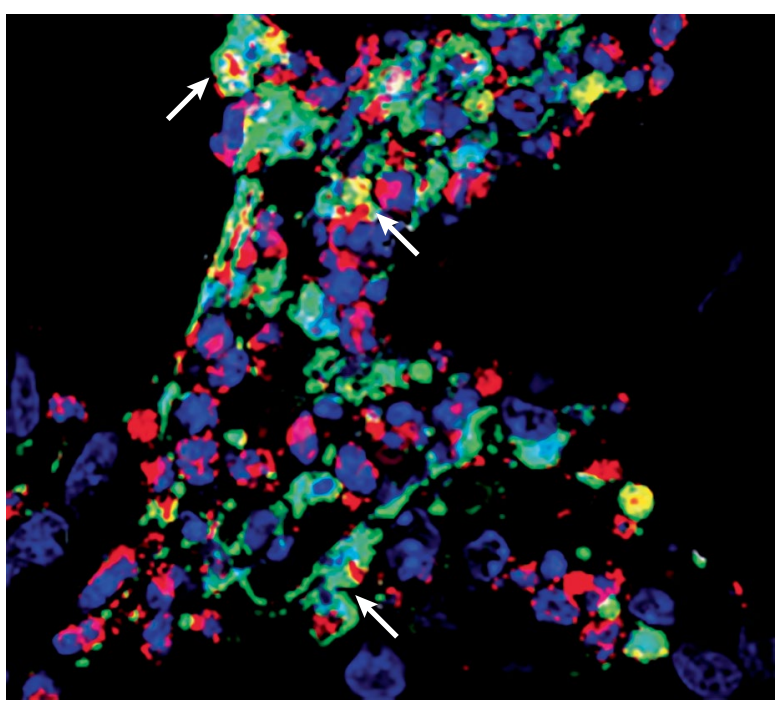

Credit: Immunofluorescence image of human liver metastases showing presence of NETs. White arrows indicate NETs, myeloperoxidase is red, citrullinated histone $\mathrm{H} 3$ is green and nuclei are stained blue. Adapted from Yang et al., Springer Nature Limited. role in trapping microorganisms, the proposed mechanism for NET-associated metastasis was presumed to involve the trapping of disseminated cancer cells. However, whether NETs are present in human cancers is largely unknown. In the latest study, a group of investigators sought to examine the functional role of NETs in liver metastasis.

First, using samples of primary tumours and metastatic lesions, as well as serum, from patients with colon cancer and breast cancer, the researchers showed that markers of NETs and their formation were readily detected in metastases but were scarce in the primary tumours. Metastatic lesions in the liver exhibited the most abundant NET infiltration and serum levels of NETs were also higher in patients with liver metastases than in those without metastases or with metastases in other organs. Using animal models injected with colon cancer or breast cancer cells, the team also showed that hepatic NETs were generated from infiltrating neutrophils before metastases could be detected and that circulating NETs might originate from the liver.

To confirm a role for NETs in liver metastasis in vivo, tumour cells were injected into mice deficient for peptidylarginine deiminase 4 (PAD4), which is a key enzyme in the formation of NETs. Compared with wild-type mice, liver metastases were significantly reduced in PAD4-deficient mice. "Our data suggest that NETs have an essential role in the cascade by which liver metastases are formed," write the authors.

On the basis of observations showing NETs in both serum and liver metastases of patients and mice, the researchers tested the hypothesis that NET-DNA might attract metastatic cancer cells. Using human cell lines, NET-DNA was found to substantially promote the migration and adhesion of cancer cells, indicating that NET-DNA does act as a chemotactic factor. Examining cell surface proteins of cancer cells led to the identification of the transmembrane protein CCDC25 as a potential receptor for this interaction.

Knockout of CCDC25 in a human colon cancer cell line abrogated in vitro cytoskeleton remodelling induced by NET-DNA and reduced chemotaxis towards NET-DNA. Furthermore, injection of CCDC25-deficient cancer cells into mice inhibited the formation of liver metastases. "These results indicate that NET-DNA induces migration, adhesion and proliferation of tumour cells via interaction with CCDC25," write the authors.

Finally, going back to their cohorts of patients with breast cancer and colon cancer, the investigators noted clear membrane staining of CCDC25 at the invasive front of tumours, and higher levels of CCDC25 were associated with reduced long-term survival. They also found that serum levels of NETs could predict the occurrence of liver metastases in patients with early-stage breast cancer. "Increased levels of NETs in blood could act as a biomarker to specifically predict the long-term risk of liver metastases," write the authors. As targeting of CCDC25 reduced the formation of NET-mediated metastases in mouse models, this protein could be a promising therapeutic target for metastasis.

Iain Dickson

ORIGINAL ARTICLE Yang, L. et al. DNA of neutrophil extracellular traps promotes cancer metastasis via CCDC25. Nature 583, 133-138 (2020)

RELATED ARTICLE Honda, M. \& Kubes, P. Neutrophils and neutrophil extracellular traps in the liver and gastrointestinal system. Nat. Rev. Gastroenterol. Hepatol. 15, 206-221 (2018) 